\title{
Co-Therapeut Pferd
}

\section{Franziska Burkhardt}

Dr. med., Fachärztin für Psychiatrie und Psychotherapie, Mitglied FMH

\author{
Seit Jahren arbeite ich mit komplextraumatisierten Menschen psychotherapeu- \\ tisch unter Einbezug unserer Pferde. Den Mut, meine psychotherapeutische Ausbil- \\ dung mit meiner jahrzehntelangen Erfahrung mit Pferden zu verbinden, gab mir \\ damals das Bewusstwerden, dass bei diesen Menschen ein wichtiger Teil der Psy- \\ chotherapie darin besteht, so Gefühle wie Vertrauen in sich und andere, Freude, \\ Sicherheit überhaupt einmal erfahren zu können.
}

Das Herdentier Pferd, welches hauptsächlich über die Körpersprache kommuniziert, reagiert unmittelbar und äusserst sensitiv auf die inneren Vorgänge bei seinem Partner. Seine Muskelspannung, Atemfrequenz, sein Pulsschlag und damit sein emotioneller Zustand schwingen synchron mit den entsprechenden Veränderungen bei seinem Partner Mensch mit. So hat mich mein Pferd bei einem Ritt im Sturm mit Glatteis gelehrt, dass anscheinend gewisse Gedanken eine unmittelbare und tiefere Entspannung bringen als zum Beispiel bewusstes Atmen. An einer gewissen Stelle kam in mir damals automatisch der Gedanke «jetzt bisch grettet» hoch. Praktisch zeitgleich tat mein Reitpferd einen tiefen Entspannungsatemzug. Gedankenlesen kann mein Pferd nicht. Aber es kann meinen Körper wahrscheinlich besser lesen als ich selber. Also

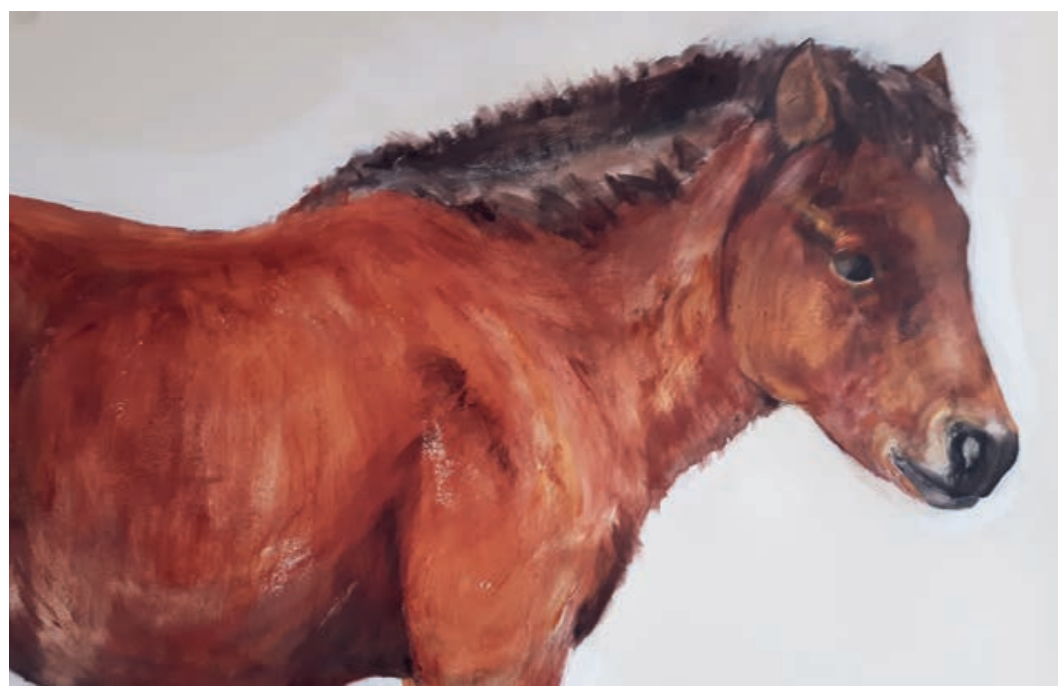

Franziska Burkhardt

Das Fohlen (Ausschnitt)

$140 \times 125 \mathrm{~cm}$, Acryl auf Hadernpapier, 2017. habe ich den Gedanken nochmals gedacht und mich beobachtet. Und siehe, meine Atmung entspannte sich reflexartig. Wunderbares Therapiematerial. Die Patientin führt das Pferd. Dieses atmet plötzlich tief aus. Frage: Oh, ihr Pferd hat gerade tief ausgeatmet und entspannt, an was haben Sie gerade gedacht? Wenn ich dann noch meine Sturmrittgeschichte erzähle, ist den Patienten sofort klar, was ich meine. «Wir haben es geschafft", «ich habe gar nichts gedacht», können dann Antworten sein. Damit lernen sie ihre unterschiedliche Körperspannung wahrzunehmen und dass diese mit ihren Gedanken zusammenhängen kann, sie also nicht nur von aussen abhängig sind, sondern sich selber auch beeinflussen können. Dasselbe gilt natürlich auch für andere Gefühle. Zeigt der Co-Therapeut Pferd Stresszeichen, stellt sich die spannende Frage, hat er sich wegen eines Raschelns im Gebüsch erschreckt, möchte er endlich seinen Hafer, bin ich, der Herdenboss, angespannt (das heisst: Auch ich muss mich mit meinen Gefühlen einbringen), oder eben, hat es mit der führenden Patientin zu tun. So können die Patienten sehr indirekt und freilassend auf ihre Gefühle angesprochen werden. Da sie in einer entspannten und freudvollen Atmosphäre sind, die Pferde lieben und mit ihnen gerecht sein möchten, kommen immer sehr ehrliche Antworten über ihren momentanen Zustand. Ich bedanke mich dann auch bei ihnen, da ich letztendlich durch ihre Offenheit mehr über das Zusammenspiel Pferd-Mensch erfahren darf, was wiederum anderen Patienten weiterhelfen kann.

Dass Co-Therapeut Pferd seinen Job gut machen kann, müssen gewisse Bedingungen erfüllt sein. Eine artgerechte Haltung und regelmässige Bewegung gehören genauso dazu, wie eine entsprechende Ausbildung. Bei der Form, wie ich mit den Pferden arbeite, ist es wichtig, dass ich als Herdenboss für das Pferd immer 
präsent bin und ihm dadurch die innere wie auch die äussere Sicherheit gebe. In diese Atmosphäre ist natürlich auch die Patientin eingebettet.

Ein erstaunliches Beispiel dazu zeigte mein Leibpferd. Die Patientin führte das Pferd im Gelände voraus. Ich ging mit dem zweiten Pferd zirka sieben Meter hinter den beiden und konzentrierte mich mental auf das vordere Team, um dort zu stützen. Da alles gut ging, dachte ich, das Pferd neben mir hätte auch etwas Konzentration verdient, und wendete mich innerlich vermehrt diesem zu. Unmittelbar darauf blieb das vorne laufende Therapiepferd stehen und schaute $\mathrm{zu}$ mir zurück.

Mit Patientinnen, welche noch keinen Kontakt mit Pferden hatten, arbeite ich vom Boden aus. Nach ausgiebigem Streicheln und «Putzmassieren" gehen wir spazieren oder üben auf einem Platz mit einem einfachen Parcours. Dazu heisst es einmal, das Pferd in Gang zu setzen, zu aktivieren, wie es in der Fachspra-

\section{Von wo kriegt man positive Vorwärtsenergie, wenn man ständig im inneren Rückzug und mit sich am Hadern ist?}

che so schön heisst. Nach all dem Kuscheln sind alle ganz entspannt. Leicht am Strick gezupft und dabei das Pferd liebevoll angestrahlt. Reaktion: Das Pferd schaut liebevoll zurück (es spricht ja die Körpersprache), macht aber keinen Schritt. Jetzt heisst es erst einmal, sich selber zu aktivieren und genau zu wissen, wohin man will. Von wo kriegt man positive Vorwärtsenergie, wenn man ständig im inneren Rückzug und mit sich am Hadern ist? (Hier werden übrigens auch diagnostische Elemente oft viel rascher sichtbar als in der Praxis.) Einer Patientin, die sich lange bemühte, kam plötzlich der rettende Gedanke; sie versetzte sich in die Fasnachtscliquen-Marschstimmung, stellte sich den Tambourmajor vor, und siehe da, Co-Therapeut Pferd marschierte los und folgte ihr flüssig in allen Übungen. Den Weg zeigt man dem Pferd, indem man diesen deutlich und konzentriert geht. Eine Patientin führte im Wald wunderbar flüssig voraus, an einer Kreuzung sollte sie rechts abbiegen. Wie auf einer Verkehrsstrasse dreht sie sich erst einmal nach links, um zu schauen, ob etwas kommt, biegt aber gleichzeitig wie geplant nach rechts ab. Ihr Pferd bleibt stehen. Jetzt wird die Patientin einmal kräftig gelobt: «Das Pferd ist mit Ihrer Körperdrehung nach links wunderbar mitgekommen, das heisst, es ist mit Ihnen als seiner Führerin bestens verbunden. Es konnte dann das zeitgleiche Nachrechts-Gehen nicht einordnen, deshalb blieb es stehen. Jetzt dürfen Sie das Pferd einmal loben, unser Ziel ist ja, die Verbindung mit dem Pferd aufnehmen zu können, und nicht der erfolgreich absolvierte Parcours.» Eine andere Patientin getraute sich nicht, ihr Pferd von dessen Lieblingsbeschäftigung, dem Grasfressen, abzuhalten. Nach einigen Stunden klappte der ganze Spaziergang ohne eine Fresseinlage. Die Patientin strahlte: «Ich hab es geschafft, und die Luna [ihr Pferd] hat mich trotzdem noch gerne.» Der Anfang einer Neuerfahrung bei einer Bindungsstörung. Eine andere Patientin hatte mit ihrem Pferd sehr gut gearbeitet. Da die Konzentration von beiden natürlicherweise nachlässt, stoppen wir in diesem guten Moment. Plötzlich sagt sie: «Jetzt spüre ich komischerweise genau das gleiche schwierige und beklemmende Gefühl, welches immer bei der Arbeit auftritt. Dabei liebe ich es ja so, mit den Pferden etwas machen zu können und etwas zu lernen.» Mittendrin sind wir in ihrem zentralen Thema.

Mit Patientinnen, welche eine langjährige Reiterfahrung mitbringen, finden psychotherapeutische Gespräche beim Reiten statt. So ist neben allem Schweren auch immer etwas Freudvolles vorhanden, und wie bekannt ist, hilft eine freudigere Einstellung beim Erlernen von Neuem. Wird die Patientin von negativen Gefühlen überflutet, hat man zudem jederzeit ein wirksames Mittel der Reorientierung zur Hand, in dem ich ganz einfach frage: «Spüren Sie Ihr Pferd noch? Wollen wir kurz traben oder galoppieren, damit Sie sich wieder auf das Pferd konzentrieren können?» Für viele dieser Patientinnen war das Pferd in der Kindheit unter Umständen das einzig Positive, und so werden diese guten Kindheitsgefühle durch das Pferd wieder aktiviert und können wesentlich zur Besserung beitragen.

Bei beiden Therapieformen biete ich dadurch, dass ich die Verantwortung als "Herdenleiterin» übernehme, das heisst mich mit der Patientin und ihrem Pferd beobachtend verbinde, die Atmosphäre eines Getragenwerdens, ähnlich jener, die zwischen einer Mutter und ihrem Kind im Idealfall besteht.

Durch das gemeinsame Tun entsteht viel rascher Vertrauen als in der Praxisatmosphäre. Zudem sind die Patienten nicht nur mit ihren Schwächen, sondern auch mit ihren Fähigkeiten in die therapeutische Beziehung eingebunden.

Indem ich ausspreche, was ich am Pferd beobachte, ist die Patientin freier, über ihren seelischen Zustand zu sprechen.

Es ist eine erfüllende Arbeit, bei der ich, dank der Offenheit meiner Patienten, immer mehr über das Zusammenspiel von Co-Therapeut Pferd und seinem Menschen verstehen und lernen darf.

Letztendlich aber ist das Pferd an sich, wie andere Tiere auch, für viele Menschen heilsam. 